\title{
On the CFT duals for near-extremal black holes
}

\author{
Jørgen Rasmussen \\ Department of Mathematics and Statistics, University of Melbourne \\ Parkville, Victoria 3010, Australia \\ j.rasmussen@ms.unimelb.edu.au
}

\begin{abstract}
We consider Kerr-Newman-AdS-dS black holes near extremality and work out the near-horizon geometry of these near-extremal black holes. We identify the exact $U(1)_{L} \times U(1)_{R}$ isometries of the near-horizon geometry and provide boundary conditions enhancing them to a pair of commuting Virasoro algebras. The conserved charges of the corresponding asymptotic symmetries are found to be well defined and non-vanishing and to yield central charges $c_{L} \neq 0$ and $c_{R}=0$. The Cardy formula subsequently reproduces the Bekenstein-Hawking entropy of the black hole. This suggests that the near-extremal Kerr-Newman-AdS-dS black hole is holographically dual to a non-chiral two-dimensional conformal field theory.
\end{abstract}




\section{Introduction}

In the seminal work [1, quantum gravity on three-dimensional anti-de Sitter (AdS) space was found to be holographically dual to a two-dimensional conformal field theory (CFT). The Kerr/CFT correspondence [2] extends this duality by asserting that quantum theory on the near-horizon geometry of the extremal four-dimensional Kerr black hole [3] is holographically dual to a chiral CFT in two dimensions. This near-horizon geometry has a $U(1)_{L} \times S L(2, \mathbb{R})_{R}$ isometry group and is called the NHEK geometry, and using the formalism of [4], it was found [2] that certain boundary conditions enhance the $U(1)_{L}$ symmetry to a Virasoro algebra. The consistency of these boundary conditions was subsequently confirmed in [5, 6], while a partial classification of boundary conditions with the same properties was provided in [7]. Boundary conditions enhancing the $S L(2, \mathbb{R})_{R}$ isometries to a Virasoro algebra were examined in [8, 7]. Strong evidence [2] for the Kerr/CFT correspondence is found in the exact agreement between the macroscopic BekensteinHawking entropy [9] of the black hole and the Cardy formula for the dual CFT entropy. This analysis has been successfully generalized and applied to a variety of extremal black holes [10, 11, 12, 13, 5, 6, 14]. The extension to the near-horizon geometry of the extremal Kerr-Newman-AdS-dS black hole [15, 16], in particular, is worked out in [16].

Extending the Kerr/CFT correspondence to the near-extremal Kerr black hole, however, has presented some serious challenges. The dual two-dimensional CFT should be non-chiral and hence accommodate two mutually commutative Virasoro algebras. Based on a variety of approaches, several insightful results on these near-extremal black holes have been obtained [17. Extending the approach of [18], in particular, the work [19] on black-hole superradiance and the subsequent generalizations thereof [20] have provided highly non-trivial support for the Kerr/CFT correspondence. These results, however, are all based on deviations from the geometrical approach of [2] since consistent boundary conditions which allow for both left- and right-moving sectors have been identified only very recently [21]. Another very recent development is the analysis of the so-called hidden conformal symmetry [22] of the Kerr black hole. It is noted that this symmetry is not a symmetry of the spacetime geometry.

It is the main objective of the present work to extend the work [21] on the near-horizon geometry of the near-extremal Kerr black hole, the so-called near-NHEK geometry, to the nearhorizon geometry of the near-extremal Kerr-Newman-AdS-dS black hole using the results [16] on the extremal case. We work out the metric of this near-horizon geometry, identify its exact $U(1)_{L} \times U(1)_{R}$ isometry group, and provide boundary conditions enhancing these symmetries to a pair of commuting Virasoro algebras. The conserved charges of the corresponding asymptotic symmetries are found to be well defined and non-vanishing and to yield central charges $c_{L} \neq 0$ and $c_{R}=0$. The Cardy formula subsequently reproduces the Bekenstein-Hawking entropy of the black hole. This supports the assertion that the near-extremal Kerr-Newman-AdS-dS black hole is holographically dual to a non-chiral two-dimensional CFT. Here we only consider the geometry of the Kerr-Newman-AdS-dS black hole but hope to address elsewhere the inclusion of the gauge field.

It is noted that the Virasoro algebra has been observed in other black-hole contexts as well. Extending the work [23] on the entropy of three-dimensional black holes such as the BTZ black hole [24], it was found in [25, 26] that a copy of the Virasoro algebra appears in the near-horizon region of any black hole and that it reproduces the black-hole entropy using the Cardy formula. 


\section{Kerr-Newman-AdS-dS black hole}

\subsection{Geometry}

In Boyer-Lindquist-type coordinates, and using the unit convention where $G=\hbar=c=1$, a Kerr-Newman-AdS-dS black hole [15, 16] is described by

$$
d \hat{s}^{2}=-\frac{\Delta_{r}}{\rho^{2}}\left(d \hat{t}-\frac{a}{\Xi} \sin ^{2} \theta d \hat{\phi}\right)^{2}+\frac{\rho^{2}}{\Delta_{r}} d \hat{r}^{2}+\frac{\rho^{2}}{\Delta_{\theta}} d \theta^{2}+\frac{\Delta_{\theta}}{\rho^{2}} \sin ^{2} \theta\left(a d \hat{t}-\frac{\hat{r}^{2}+a^{2}}{\Xi} d \hat{\phi}\right)^{2}
$$

where

$$
\begin{aligned}
& \Delta_{r}=\left(\hat{r}^{2}+a^{2}\right)\left(1+\frac{\hat{r}^{2}}{\ell^{2}}\right)-2 M \hat{r}+q^{2}, \quad q^{2}=q_{e}^{2}+q_{m}^{2} \\
& \Delta_{\theta}=1-\frac{a^{2}}{\ell^{2}} \cos ^{2} \theta, \quad \rho^{2}=\hat{r}^{2}+a^{2} \cos ^{2} \theta, \quad \Xi=1-\frac{a^{2}}{\ell^{2}}
\end{aligned}
$$

This metric is parameterized by the ADM mass $M_{A D M}=M / \Xi^{2}$, angular momentum $J=a M / \Xi^{2}$ and electric and magnetic charges $Q_{e}=q_{e} / \Xi$ and $Q_{m}=q_{m} / \Xi$, and it is AdS (dS) for positive (negative) renormalized cosmological constant $\ell^{-2}$. It reduces to Kerr-Newman for $\ell^{-2}=0$ and further to Kerr for $q^{2}=0$. The horizons are located at the positive zeros of $\Delta_{r}$, and the value of $\hat{r}$ at the outer horizon is denoted by $r_{+}$. The Hawking temperature $T_{H}$, angular velocity $\Omega_{H}$ of the event horizon, and entropy $S$ are given by

$$
T_{H}=\frac{r_{+}\left(1+\frac{a^{2}}{\ell^{2}}+\frac{3 r_{+}^{2}}{\ell^{2}}-\frac{a^{2}+q^{2}}{r_{+}^{2}}\right)}{4 \pi\left(r_{+}^{2}+a^{2}\right)}, \quad \Omega_{H}=\frac{a \Xi}{r_{+}^{2}+a^{2}}, \quad S=\frac{\pi\left(r_{+}^{2}+a^{2}\right)}{\Xi}
$$

For later convenience, we follow [16] and introduce

$$
r_{0}^{2}=\frac{\left(r_{+}^{2}+a^{2}\right)\left(1-\frac{r_{+}^{2}}{\ell^{2}}\right)}{1+\frac{6 r_{+}^{2}}{\ell^{2}}-\frac{3 r_{+}^{4}}{\ell^{4}}-\frac{q^{2}}{\ell^{2}}}, \quad \rho_{+}^{2}=r_{+}^{2}+a^{2} \cos ^{2} \theta
$$

and

$$
\Gamma(\theta)=\frac{\rho_{+}^{2} r_{0}^{2}}{r_{+}^{2}+a^{2}}, \quad \alpha(\theta)=\frac{r_{+}^{2}+a^{2}}{\Delta_{\theta} r_{0}^{2}}, \quad \gamma(\theta)=\frac{\Delta_{\theta} \sin ^{2} \theta\left(r_{+}^{2}+a^{2}\right)^{2}}{\Xi^{2} \rho_{+}^{2}}, \quad k=\frac{2 a \Xi r_{0}^{2} r_{+}}{\left(r_{+}^{2}+a^{2}\right)^{2}}
$$

\subsection{Extremal black holes}

When the inner and outer horizons coalesce, the black hole is said to be extremal. This happens when the otherwise single pole $r_{+}$of $\Delta_{r}$ is a double pole in which case

$$
\Delta_{r}\left(r_{+}\right)=\Delta_{r}^{\prime}\left(r_{+}\right)=0
$$

We denote the value of $\hat{r}$ at this single horizon by $\bar{r}$ and use the related notation $\bar{a}, \bar{T}_{H}$ and $\bar{\Omega}_{H}$, in particular, for the evaluation of the various parameters at extremality. The conditions (2.6) can be used to express $M$ and $\bar{a}^{2}$ in terms of $\bar{r}$

$$
M=\frac{\bar{r}\left[\left(1+\frac{\bar{r}^{2}}{\ell^{2}}\right)^{2}-\frac{q^{2}}{\ell^{2}}\right]}{1-\frac{\bar{r}^{2}}{\ell^{2}}}, \quad \bar{a}^{2}=\frac{\bar{r}^{2}\left(1+\frac{3 \bar{r}^{2}}{\ell^{2}}\right)-q^{2}}{1-\frac{\bar{r}^{2}}{\ell^{2}}}
$$

and we readily recover $\bar{T}_{H}=0$. 


\section{$2.3 \quad$ Near-horizon geometry of extremal black holes}

To describe the near-horizon geometry of the extremal Kerr-Newman-AdS-dS black hole, we follow [3, 16] and introduce the new coordinates $t, r, \phi$ where

$$
\hat{t}=\frac{t \bar{r}_{0}}{\epsilon}, \quad \hat{r}=\bar{r}+\epsilon \bar{r}_{0} r, \quad \hat{\phi}=\phi+\frac{\bar{\Omega}_{H} \bar{r}_{0} t}{\epsilon}
$$

The near-horizon geometry is obtained by taking the limit $\epsilon \rightarrow 0$ in which case the metric (2.1) becomes

$$
d \bar{s}^{2}=\bar{\Gamma}(\theta)\left(-r^{2} d t^{2}+\frac{d r^{2}}{r^{2}}+\bar{\alpha}(\theta) d \theta^{2}\right)+\bar{\gamma}(\theta)(d \phi+\bar{k} r d t)^{2}
$$

The exact isometry group of the near-horizon geometry (2.9) is $U(1)_{L} \times S L(2, \mathbb{R})_{R}$ and it is generated by

$$
\left\{\partial_{\phi}\right\} \cup\left\{\partial_{t}, t \partial_{t}-r \partial_{r},\left(t^{2}+\frac{1}{r^{2}}\right) \partial_{t}-2 t r \partial_{r}-\frac{2 \bar{k}}{r} \partial_{\phi}\right\}
$$

\section{$2.4 \quad$ Near-extremal geometry}

We are interested in infinitesimal excitations above extremality of the Kerr-Newman-AdS-dS black

hole. To describe this near-extremal geometry, we consider the situation where the outer horizon $r_{+}$is infinitesimally larger than the extremal horizon $\bar{r}$

$$
r_{+}=\bar{r}+\epsilon \bar{\kappa}
$$

Here $\bar{\kappa}$ is expressed in terms of the parameters of the extremal black hole, while $\epsilon$ is an infinitesimal parameter whose limit $\epsilon \rightarrow 0$ we are ultimately interested in, where

$$
a^{2}=\bar{a}^{2}-\frac{\epsilon^{2} \bar{\kappa}^{2}\left(\bar{r}^{2}+\bar{a}^{2}\right)}{\bar{r}_{0}^{2}\left(1+\frac{\bar{r}^{2}}{\ell^{2}}\right)}+\mathcal{O}\left(\epsilon^{3}\right), \quad T_{H}=\frac{\epsilon \bar{\kappa}}{2 \pi \bar{r}_{0}^{2}}+\mathcal{O}\left(\epsilon^{2}\right), \quad \Omega_{H}=\bar{\Omega}_{H}\left(1-\frac{2 \epsilon \bar{\kappa} \bar{r}}{\bar{r}^{2}+\bar{a}^{2}}\right)+\mathcal{O}\left(\epsilon^{2}\right)
$$

Following [11, we introduce

$$
T_{L}=\lim _{r_{+} \rightarrow \bar{r}} \frac{T_{H}}{\bar{\Omega}_{H}-\Omega_{H}}=\frac{1}{2 \pi \bar{k}}
$$

and interpret it as the Frolov-Thorne temperature [27] associated with the azimuthal angle $\phi$. Although the asymptotically-defined Hawking temperature $T_{H}$ vanishes in the limit $\epsilon \rightarrow 0$, we can use that $T_{H} / \epsilon$ is non-vanishing and finite to introduce

$$
T_{R}=\lim _{r_{+} \rightarrow \bar{r}} \frac{r_{0}^{2} T_{H}}{r_{+} \epsilon}=\frac{\bar{\kappa}}{2 \pi \bar{r}}
$$

and interpret it as a temperature associated with the near-extremality of the geometry.

\subsection{Near-horizon geometry of near-extremal black holes}

To describe the near-horizon geometry of the near-extremal Kerr-Newman-AdS-dS black hole, we use the coordinates $t, r, \phi$ where

$$
\hat{t}=\frac{\bar{r}_{0}^{2} t}{\epsilon}, \quad \hat{r}=\bar{r}+\epsilon(r+\lambda \bar{\kappa}), \quad \hat{\phi}=\phi+\frac{\bar{\Omega}_{H} \bar{r}_{0}^{2} t}{\epsilon}
$$


and where we have introduced the additional parameter $\lambda$. In the limit $\epsilon \rightarrow 0$, we find

$$
\Delta_{r}=\frac{\epsilon^{2}\left(\bar{r}^{2}+\bar{a}^{2}\right)}{\bar{r}_{0}^{2}}(r+(\lambda-1) \bar{\kappa})(r+(\lambda+1) \bar{\kappa})+\mathcal{O}\left(\epsilon^{3}\right)
$$

and hence

$$
\begin{aligned}
d s^{2} & =\Gamma(\theta)\left[-(r+(\lambda-1) \bar{\kappa})(r+(\lambda+1) \bar{\kappa}) d t^{2}+\frac{d r^{2}}{(r+(\lambda-1) \bar{\kappa})(r+(\lambda+1) \bar{\kappa})}+\alpha(\theta) d \theta^{2}\right] \\
& +\gamma(\theta)[d \phi+k(r+\lambda \bar{\kappa}) d t]^{2}
\end{aligned}
$$

For all $\lambda$, this is invariant under the action of the exact isometry group $U(1)_{L} \times U(1)_{R}$ generated by

$$
\left\{\partial_{\phi}\right\} \cup\left\{\partial_{t}\right\}
$$

These isometries commute with translations in $r$ generated by $\partial_{r}$ and realized by varying $\lambda$. We note that the determinant of the metric (2.17) only depends on $\theta$ as

$$
\sqrt{-g}=\sqrt{\Gamma^{3}(\theta) \alpha(\theta) \gamma(\theta)}=\frac{\sin \theta \rho_{+}^{2} r_{0}^{2}}{\Xi}
$$

In the following, focus will be on $\lambda=1$ in which case

$$
r=\frac{\hat{r}-r_{+}}{\epsilon}
$$

and

$$
\left.d s^{2}\right|_{\lambda=1}=\Gamma(\theta)\left(-r(r+2 \bar{\kappa}) d t^{2}+\frac{d r^{2}}{r(r+2 \bar{\kappa})}+\alpha(\theta) d \theta^{2}\right)+\gamma(\theta)(d \phi+k(r+\bar{\kappa}) d t)^{2}
$$

As above, special cases are obtained by setting $\ell^{-2}=0$ or $q^{2}=0$. The near-NHEK geometry [13, 5, 19, 21, in particular, follows when both of these conditions apply. We note that the coordinate convention for the near-NHEK geometry used in [19, 21] differs from the one following from (2.15), but is obtained by the replacement $t, r \rightarrow t / M, M r$.

\section{Holographically dual CFT}

\subsection{Boundary conditions and conserved charges}

We are interested in perturbations $h_{\mu \nu}$ of the near-horizon geometry (2.21) of the near-extremal Kerr-Newman-AdS-dS black hole. Following [21] on the near-NHEK geometry, we introduce the fall-off conditions

$$
h_{\mu \nu}=\mathcal{O}\left(\begin{array}{cccc}
r^{2} & r^{-3} & r^{1} & r^{-2} \\
& r^{-4} & r^{-1} & r^{-3} \\
& & 1 & r^{-2} \\
& & & r^{-2}
\end{array}\right), \quad h_{\mu \nu}=h_{\nu \mu}
$$

in addition to a couple of supplementary conditions to be discussed below. Asymptotic symmetries are generated by the diffeomorphisms whose action on the metric generates metric fluctuations 
compatible with the full set of boundary conditions. Initially focusing on the fall-off conditions (3.1), we find the asymptotic Killing vectors

$$
\begin{aligned}
& K_{\epsilon}=\left[\mathcal{O}\left(r^{-4}\right)\right] \partial_{t}+\left[-(r+\bar{\kappa}) \epsilon^{\prime}(\phi)+\mathcal{O}\left(r^{-1}\right)\right] \partial_{r}+\left[\epsilon(\phi)+\mathcal{O}\left(r^{-3}\right)\right] \partial_{\phi}+\left[\mathcal{O}\left(r^{-2}\right)\right] \partial_{\theta} \\
& \mathcal{K}_{\varepsilon}=\left[\varepsilon(t)+\mathcal{O}\left(r^{-4}\right)\right] \partial_{t}+\left[\mathcal{O}\left(r^{-1}\right)\right] \partial_{r}+\left[\mathcal{O}\left(r^{-3}\right)\right] \partial_{\phi}+\left[\mathcal{O}\left(r^{-2}\right)\right] \partial_{\theta}
\end{aligned}
$$

where $\epsilon(\phi)$ and $\varepsilon(t)$ are smooth functions. The generators of the corresponding asymptotic symmetries thus read

$$
\xi=-(r+\bar{\kappa}) \epsilon^{\prime}(\phi) \partial_{r}+\epsilon(\phi) \partial_{\phi}, \quad \zeta=\varepsilon(t) \partial_{t}
$$

and form a commuting pair of centreless Virasoro algebras

$$
\left[\xi_{\epsilon}, \xi_{\hat{\epsilon}}\right]=\xi_{\epsilon \hat{\epsilon}^{\prime}-\epsilon^{\prime} \hat{\epsilon}}, \quad\left[\zeta_{\varepsilon}, \zeta_{\hat{\varepsilon}}\right]=\zeta_{\varepsilon \hat{\varepsilon}^{\prime}-\varepsilon^{\prime} \hat{\varepsilon}}, \quad\left[\xi_{\epsilon}, \zeta_{\varepsilon}\right]=0
$$

Along $\xi$ and $\zeta$, the Lie derivatives of the near-horizon geometry (2.21) are worked out to be

$$
\begin{aligned}
& \mathcal{L}_{\xi} g_{\mu \nu}=\left(\begin{array}{cccc}
2\left(\Gamma-k^{2} \gamma\right)(r+\bar{\kappa})^{2} \epsilon^{\prime}(\phi) & 0 & 0 & 0 \\
0 & \frac{2 \bar{\kappa}^{2} \Gamma \epsilon^{\prime}(\phi)}{r^{2}(r+2 \bar{\kappa})^{2}} & -\frac{\Gamma(r+\bar{\kappa}) \epsilon^{\prime \prime}(\phi)}{r(r+2 \bar{\kappa})} & 0 \\
0 & -\frac{\Gamma(r+\bar{\kappa}) \epsilon^{\prime \prime}(\phi)}{r(r+2 \bar{\kappa})} & 2 \gamma \epsilon^{\prime}(\phi) & 0 \\
0 & 0 & 0 & 0
\end{array}\right) \\
& \mathcal{L}_{\zeta} g_{\mu \nu}=\varepsilon^{\prime}(t)\left(\begin{array}{cccc}
-2 \Gamma r(r+2 \bar{\kappa})+2 k^{2} \gamma(r+\bar{\kappa})^{2} & 0 & k \gamma(r+\bar{\kappa}) & 0 \\
0 & 0 & 0 & 0 \\
k \gamma(r+\bar{\kappa}) & 0 & 0 & 0 \\
0 & 0 & 0 & 0
\end{array}\right)
\end{aligned}
$$

where we have introduced the abbreviations

$$
\Gamma=\Gamma(\theta), \quad \alpha=\alpha(\theta), \quad \gamma=\gamma(\theta)
$$

To an asymptotic symmetry generator $\eta$, one associates [4] the conserved charge

$$
Q_{\eta}=\frac{1}{8 \pi} \int_{\partial \Sigma} \sqrt{-g} k_{\eta}[h ; g]=\frac{1}{8 \pi} \int_{\partial \Sigma} \frac{\sqrt{-g}}{4} \epsilon_{\alpha \beta \mu \nu} d_{\eta}^{\mu \nu}[h ; g] d x^{\alpha} \wedge d x^{\beta}
$$

where

$$
d_{\eta}^{\mu \nu}[h ; g]=\eta^{\nu} D^{\mu} h-\eta^{\nu} D_{\sigma} h^{\mu \sigma}+\eta_{\sigma} D^{\nu} h^{\mu \sigma}-h^{\nu \sigma} D_{\sigma} \eta^{\mu}+\frac{1}{2} h D^{\nu} \eta^{\mu}+\frac{1}{2} h^{\sigma \nu}\left(D^{\mu} \eta_{\sigma}+D_{\sigma} \eta^{\mu}\right)
$$

and where $\partial \Sigma$ is the boundary of a three-dimensional spatial volume near spatial infinity. Indices are lowered and raised using the background metric $g_{\mu \nu}$ and its inverse, $D_{\mu}$ denotes a background covariant derivative, while $h$ is defined as $h=g^{\mu \nu} h_{\mu \nu}$. To be a well-defined charge in the asymptotic limit, the underlying integral must be finite as $r \rightarrow \infty$. If the charge vanishes, the asymptotic symmetry is rendered trivial. The asymptotic symmetry group is then generated by the diffeomorphisms whose charges are well-defined and non-vanishing. The algebra generated by the set of well-defined charges is governed by the Dirac brackets computed [4] as

$$
\left\{Q_{\eta}, Q_{\hat{\eta}}\right\}=Q_{[\eta, \hat{\eta}]}+\frac{1}{8 \pi} \int_{\partial \Sigma} \sqrt{-g} k_{\eta}\left[\mathcal{L}_{\hat{\eta}} g ; g\right]
$$


where the integral yields the eventual central extension.

Now, considering the asymptotic $r$-expansion of $k_{\partial_{t}}[h ; g]$, we find that its divergent term (linear in $r$ ) is independent of $\bar{\kappa}$ and that its constant term (independent of $r$ ) is at most linear in $\bar{\kappa}$. Subleading terms in $r$ can be ignored as $r \rightarrow \infty$. We thus follow [21] and impose the condition

$$
\left(1-\bar{\kappa} \partial_{\bar{\kappa}}\right) Q_{\partial_{t}}=0
$$

where $\partial_{\bar{\kappa}}$ is a formal derivative in $\bar{\kappa}$. Furthermore, the conserved charge $Q_{\zeta}$ must be well-defined for all $\zeta$, not just for $\varepsilon(t)=1$ as in (3.10). To understand the potential complications arising when extending from $Q_{\partial_{t}}$ to general $Q_{\zeta}$, we examine the difference in their integrands and find the remarkably simple relation

$$
\left.\sqrt{-g}\left(k_{\zeta}[h ; g]-\varepsilon(t) k_{\partial_{t}}[h ; g]\right)\right|_{d \phi \wedge d \theta}=-\frac{1}{4} \sqrt{\frac{\alpha \gamma}{\Gamma}} \varepsilon^{\prime}(t)(r+\bar{\kappa}) h_{r \phi}
$$

valid for all $r$. It follows, in particular, that the divergent part of the asymptotic $r$-expansion of $k_{\zeta}[h ; g]$ matches the divergent part of $\varepsilon(t) k_{\partial_{t}}[h ; g]$ where

$$
\begin{aligned}
\left.\sqrt{-g} k_{\partial_{t}}[h ; g]\right|_{d \phi \wedge d \theta} & =\frac{1}{4} \sqrt{\frac{\alpha}{\Gamma^{3} \gamma}}\left\{-k^{2} \gamma^{2} r^{-1} h_{t t}+2 k \gamma\left[k^{2} \gamma-\Gamma r \partial_{r}\right] h_{t \phi}\right. \\
& \left.+2 \Gamma\left[\Gamma-k^{2} \gamma\right] r^{2} \partial_{\phi} h_{r \phi}+\left[2 \Gamma^{2}-k^{2} \Gamma \gamma-k^{4} \gamma^{2}\right] r h_{\phi \phi}\right\}+\mathcal{O}\left(r^{0}\right)
\end{aligned}
$$

Here we have used that $\partial_{r} h_{\phi \phi}=\mathcal{O}\left(r^{-2}\right)$. Since the right-hand side of (3.11) may violate the Jacobi identity for the Dirac brackets of the conserved charges $Q_{\zeta}$, we require that $h_{r \phi}$ is a total $\phi$-derivative on the boundary

$$
\left.h_{r \phi}\right|_{\partial \Sigma}=\partial_{\phi} H_{r \phi}
$$

where it is noted that total $\phi$-derivatives can be ignored in the computation of the conserved charge $Q_{\zeta}$. Under the constraints (3.1), (3.10) and (3.13), only perturbations $h$ preserving them and only background metrics $g$ which can be reached from the near-extremal geometry via a path of such perturbations are considered. This should ensure that $Q_{\xi}$ and $Q_{\zeta}$ are well defined.

Taking the constraint (3.10) explicitly into account, we find that $Q_{\zeta}$ can be written as

$$
Q_{\zeta}=\frac{1}{8 \pi} \int_{\partial \Sigma} \sqrt{-g} \hat{k}_{\zeta}[h ; g]
$$

where

$$
\sqrt{-g} \hat{k}_{\zeta}[h ; g]=\frac{k^{2} \bar{\kappa} \varepsilon(t)}{2} \sqrt{\frac{\alpha \gamma^{3}}{\Gamma^{3}}}\left(\frac{h_{t t}}{r^{2}}-\frac{k h_{t \phi}}{r}\right) d \phi \wedge d \theta+\ldots
$$

The dots indicate that terms not contributing to the charge (3.14) have been omitted. The conserved charge $Q_{\zeta}$ is thus non-vanishing for general boundary conditions satisfying the constraints (3.1), (3.10) and (3.13). We also find that the integrand in (3.7) for the conserved charge $Q_{\xi}$ is given by

$$
\begin{array}{r}
\sqrt{-g} k_{\xi}[h ; g]=\frac{1}{4} \sqrt{\frac{\alpha}{\Gamma^{3} \gamma}}\left(\gamma \epsilon(\phi)\left[-\frac{k \gamma h_{t t}}{r^{2}}+\frac{2\left(k^{2} \gamma-\Gamma r \partial_{r}\right) h_{t \phi}}{r}-2 k \Gamma r \partial_{\phi} h_{r \phi}-k\left(\Gamma+k^{2} \gamma\right) h_{\phi \phi}\right]\right. \\
\left.+2 \Gamma \epsilon^{\prime}(\phi)\left[k \gamma r h_{r \phi}-\frac{\Gamma \partial_{\phi} h_{t \phi}}{r}\right]+\epsilon^{\prime \prime}(\phi)\left[\frac{\Gamma^{2} h_{t \phi}}{r}\right]\right) d \phi \wedge d \theta+\ldots
\end{array}
$$

It is recalled that the near-horizon geometry of the extremal Kerr-Newman-AdS-dS black hole is obtained by setting $\bar{\kappa}=0$ in which case the conserved charge $Q_{\zeta}$ is seen to vanish. The conserved charge $Q_{\xi}$, on the other hand, is unaffected by setting $\bar{\kappa}=0$. 


\subsection{Central charges and entropy}

Following the recipe used in [2], for example, we introduce the basis

$$
\epsilon_{n}(\phi)=-e^{-i n \phi}
$$

and work out the corresponding Dirac bracket algebra (3.9). We thus find

$$
i\left\{Q_{\xi_{n}}, Q_{\xi_{m}}\right\}=(n-m) Q_{\xi_{n+m}}+\frac{c_{L}}{12} n\left(n^{2}-1+b\right) \delta_{n+m, 0}
$$

where $b$ is a constant while the central charge is given by

$$
c_{L}=3 k \int_{0}^{\pi} \sqrt{\Gamma(\theta) \alpha(\theta) \gamma(\theta)} d \theta
$$

After introducing the quantum generators

$$
L_{n}=Q_{\xi_{n}}+\frac{b c_{L}}{24} \delta_{n, 0}
$$

and performing the usual substitution $\{.,.\} \rightarrow-i[.,$.$] of Dirac brackets by quantum commutators$ (recalling that we are using the unit convention $\hbar=1$ ), the quantum charge algebra is recognized as the centrally-extended Virasoro algebra

$$
\left[L_{n}, L_{m}\right]=(n-m) L_{n+m}+\frac{c_{L}}{12} n\left(n^{2}-1\right) \delta_{n+m, 0}
$$

With the expressions (2.4) and (2.5) appearing in the near-horizon geometry (2.21) of the nearextremal Kerr-Newman-AdS-dS black hole, the central charge is worked out to be

$$
c_{L}=\frac{12 r_{+} \sqrt{\left(r_{+}^{2}+\frac{3 r_{+}^{4}}{\ell^{2}}-q^{2}\right)\left(1-\frac{r_{+}^{2}}{\ell^{2}}\right)}}{1+\frac{6 r_{+}^{2}}{\ell^{2}}-\frac{3 r_{+}^{4}}{\ell^{4}}-\frac{q^{2}}{\ell^{2}}}
$$

This is the same expression as the one for the central charge characterizing the chiral CFT dual to the near-horizon geometry of the extremal Kerr-Newman-AdS-dS black hole evaluated in [16].

As in the case of the near-NHEK geometry considered in [21], we find that the central charge $c_{R}$ associated with the conserved charges $Q_{\zeta}$ vanishes. By evaluating $\sqrt{-g} k_{\xi}\left[\mathcal{L}_{\zeta} g ; g\right]$ and $\sqrt{-g} k_{\zeta}\left[\mathcal{L}_{\xi} g ; g\right]$ explicitly, it is also verified that there is no central charge mixing the two conformal sectors characterized by the central charges $c_{L}$ and $c_{R}$. This establishes that the two corresponding Virasoro algebras are mutually commutative and suggests that the CFT dual to the near-extremal KerrNewman-AdS-dS black hole is non-chiral. Since $c_{R}=0$, the Cardy formula for the entropy of this dual CFT is simply given by

$$
S=\frac{\pi^{2}}{3} c_{L} T_{L}=\frac{\pi\left(r_{+}^{2}+a^{2}\right)}{\Xi}
$$

This is readily recognized as the entropy (2.3) of the Kerr-Newman-AdS-dS black hole in the (near-)extremal limit.

\section{Acknowledgments}

This work is supported by the Australian Research Council. The author thanks Omar Foda for discussions. 


\section{References}

[1] J.D. Brown, M. Henneaux, Central charges in the canonical realization of asymptotic symmetries: an example from three-dimensional gravity, Commun. Math. Phys. 104 (1986) 207-226.

[2] M. Guica, T. Hartman, W. Song, A. Strominger, The Kerr/CFT correspondence, Phys. Rev. D80 (2009) 124008, arXiv:0809.4266 [hep-th].

[3] J.M. Bardeen, G.T. Horowitz, The extreme Kerr throat geometry: a vacuum analog of AdS $S_{2} \times$ $S^{2}$, Phys. Rev. D60 (1999) 104030, arXiv:hep-th/9905099.

[4] G. Barnich, F. Brandt, Covariant theory of asymptotic symmetries, conservation laws and central charges, Nucl. Phys. B633 (2002) 3-82, arXiv:hep-th/0111246; G. Barnich, G. Compère, Surface charge algebra in gauge theories and thermodynamic integrability J. Math. Phys. 49 (2008) 042901, arXiv:0708.2378 [gr-qc].

[5] A.J. Amsel, G.T. Horowitz, D. Marolf, M.M. Roberts, No dynamics in the extremal Kerr throat, JHEP 0909 (2009) 044, arXiv:0906.2376 [hep-th].

[6] O.J.C. Dias, H.S. Reall, J.E. Santos, Kerr-CFT and gravitational perturbations, JHEP 0908 (2009) 101, arXiv:0906.2380 [hep-th]; V. Balasubramanian, J. de Boer, M.M. Sheikh-Jabbari, J. Simón, What is a chiral 2d CFT? And what does it have to do with extremal black holes?, JHEP 1002 (2010) 017, arXiv:0906.3272 [hep-th]; A.J. Amsel, D. Marolf, M.M. Roberts, On the stress tensor of Kerr/CFT, arXiv:0907.5023 [hep-th].

[7] J. Rasmussen, Isometry-preserving boundary conditions in the Kerr/CFT correspondence, Int. J. Mod. Phys. A25 (2010) 1597-1613, arXiv:0908.0184 [hep-th].

[8] Y. Matsuo, T. Tsukioka, C.-M. Yoo, Another realization of Kerr/CFT correspondence, Nucl. Phys. B825 (2010) 231-241, arXiv:0907.0303 [hep-th].

[9] J.D. Bekenstein, Black holes and entropy, Phys. Rev. D7 (1973) 2333-2346; S.W. Hawking, Particle creation by black holes, Commun. Math. Phys. 43 (1975) 199-220, Erratum-ibid. 46 (1976) 206.

[10] F. Loran, H. Soltanpanahi, Near the horizon of 5D black rings, JHEP 0903 (2009) 035, arXiv:0810.2620 [hep-th]; K. Hotta, Y. Hyakutake, T. Kubota, T. Nishinaka, H. Tanida, The CFT-interpolating Black Hole in Three Dimensions, JHEP 0901 (2009) 010, arXiv:0811.0910 [hep-th]; H. Lü, J. Mei, C.N. Pope, Kerr-AdS/CFT correspondence in diverse dimensions, JHEP 0904 (2009) 054, arXiv:0811.2225 [hep-th]; T. Azeyanagi, N. Ogawa, S. Terashima, Holographic duals of Kaluza-Klein black holes, JHEP 0904 (2009) 061, arXiv:0811.4177 [hepth]; Y. Nakayama, Emerging AdS from extremally rotating NS5-branes, Phys. Lett. B673 (2009) 272-278, arXiv:0812.2234 [hep-th]; H. Isono, T.S. Tai, W.Y. Wen, Kerr/CFT correspondence and five-dimensional BMPV black holes, Int. J. Mod. Phys. A24 (2009) 5659-5668, arXiv:0812.4440 [hep-th]; T. Azeyanagi, N. Ogawa, S. Terashima, The Kerr/CFT correspondence and string theory, Phys. Rev. D79 (2009) 106009, arXiv:0812.4883 [hep-th]; J.-J. Peng, S.-Q. Wu, Extremal Kerr black hole/CFT correspondence in the five dimensional Gödel universe, Phys. Lett. B673 (2009) 216-219, arXiv:0901.0311 [hep-th]; C.-M. Chen, J.E. Wang, Holographic duals of black holes in five-dimensional minimal supergravity, arXiv:0901.0538 
[hep-th]; F. Loran, H. Soltanpanahi, 5D extremal rotating black holes and CFT duals, Class. Quant. Grav. 26 (2009) 155019, arXiv:0901.1595 [hep-th]; A.M. Ghezelbash, Kerr/CFT correspondence in the low energy limit of heterotic string theory, JHEP 0908 (2009) 045, arXiv:0901.1670 [hep-th].

[11] D.D.K. Chow, M. Cvetic, H. Lü, C.N. Pope, Extremal black hole/CFT correspondence in (gauged) supergravities, Phys. Rev. D79 (2009) 084018, arXiv:0812.2918 [hep-th].

[12] H. Lü, J. Mei, C.N. Pope, J. Vazquez-Poritz, Extremal static AdS black hole/CFT correspondence in gauged supergravities, Phys. Lett. B673 (2009) 77-82, arXiv:0901.1677 [hep-th]; G. Compère, K. Murata, T. Nishioka, Central charges in extreme black hole/CFT correspondence, JHEP 0905 (2009) 077, arXiv:0902.1001 [hep-th]; K. Hotta, Holographic RG flow dual to attractor flow in extremal black holes, Phys. Rev. D79 (2009) 104018, arXiv:0902.3529 [hep-th]; D. Astefanesi, Y.K. Srivastava, CFT duals for attractor horizons, arXiv:0902.4033 [hep-th]; M.R. Garousi, A. Ghodsi, The RN/CFT correspondence arXiv:0902.4387 [hep-th]; A.M. Ghezelbash, Kerr-Bolt spacetimes and Kerr/CFT correspondence, arXiv:0902.4662 [hepth]; C. Krishnan, S. Kuperstein, A comment on Kerr-CFT and Wald entropy, Phys. Lett. B677 (2009) 326-331, arXiv:0903.2169 [hep-th]; T. Azeyanagi, G. Compère, N. Ogawa, Y. Tachikawa, A. Terashima, Higher-derivative corrections to the asymptotic Virasoro symmetry of $4 d$ extremal black holes, Prog. Theor. Phys. 122 (2009) 355-384, arXiv:0903.4176; X.-N. Wu, Y. Tian, Extremal isolated horizon/CFT correspondence, Phys. Rev. D80 (2009) 024014, arXiv:0904.1554 [hep-th]; L.-M. Cao, Y. Matsuo, T. Tsukioka, C.-M. Yoo, Conformal symmetry for rotating D-branes, Phys. Lett. B679 (2009) 390-395, arXiv:0906.2267 [hep-th].

[13] W.-Y. Wen, Holographic descriptions of (near-)extremal black holes in five dimensional minimal supergravity, arXiv:0903.4030 [hep-th].

[14] E. Barnes, D. Vaman, C. Wu, All 4-dimensional static, spherically, symmetric, 2-charge abelian Kaluza-Klein black holes and their CFT duals, arXiv:0908.2425 [hep-th]; R. Fareghbal, A dual CFT for Schwarzschild black hole, arXiv:0908.4394 [hep-th]; V. Jejjala, S. Nampuri, Cardy and Kerr, JHEP 1002 (2010) 088, arXiv:0909.1110 [hep-th]; J. Rasmussen, A note on Kerr/CFT and free fields, to appear in Int. J. Mod. Phys. A, arXiv:0909.2924 [hep-th]; C.-M. Chen, J.-R. Sun, S.-J. Zou, The RN/CFT correspondence revisited, JHEP 1001 (2010) 057, arXiv:0910.2076 [hep-th]; D. Anninos, T. Hartman, Holography at an extremal de Sitter horizon, JHEP 1003 (2010) 096, arXiv:0910.4587 [hep-th]; H. Soltanpanahi, Near horizon of $5 D$ rotating black holes from $2 D$ perspective, arXiv:0911.0148 [hep-th]; J.J. Peng, S.Q. Wu, Extremal Kerr/CFT correspondence of five-dimensional rotating (charged) black holes with squashed horizons, Nucl. Phys. B828 (2010) 273-288, arXiv:0911.5070 [hep-th]; J. Mei, The entropy for general extremal black holes, arXiv:1002.1349 [hep-th]; L. Rodriquez, T. Yildirim, Entropy and temperature from black-hole/near-horizon-CFT duality, arXiv:1003.0026 [hep-th].

[15] B. Carter, Hamilton-Jacobi and Schrödinger separable solutions of Einstein's equations, Commun. Math. Phys. 10 (1968) 280; J.F. Plebanski, M. Demianski, Rotating, charged, and uniformly accelerating mass in general relativity, Ann. Phys. 98 (1976) 98-127; M.M. Caldarelli, G. Cognola, D, Klemm, Thermodynamics of Kerr-Newman-AdS black holes and conformal field theories, Class. Quant. Grav. 17 (2000) 399, arXiv:hep-th/9908022.

[16] T. Hartman, K. Murata, T. Nishioka, A. Strominger, CFT duals for extreme black holes, JHEP 0904 (2009) 019, arXiv:0811.4393 [hep-th]. 
[17] A. Castro, F. Larsen, Near extremal Kerr entropy from $A d S_{2}$ quantum gravity, JHEP 0912 (2009) 037, arXiv:0908.1121 [hep-th]; C.-M. Chen, Y.-M. Huang, S.-J. Zou, Holographic duals of near-extremal Reissner-Nordstrom black holes, arXiv:1001.2833 [hep-th]; B. Chen, C.-S. Chu, Real-time correlators in Kerr/CFT correspondence, arXiv:1001.3208 [hep-th]; A. Castro, C. Keeler, F. Larsen, Three dimensional origin of $A d S_{2}$ quantum gravity, arXiv:1004.0554 [hep-th].

[18] J.M. Maldacena, A. Strominger, Black hole greybody factors and D-brane spectroscopy, Phys. Rev. D55 (1997) 861-870, arXiv:hep-th/9609026 J.M. Maldacena, A. Strominger, Universal low-energy dynamics for rotating black holes, Phys. Rev. D56 (1997) 4975-4983, arXiv:hep-th/9702015.

[19] I. Bredberg, T. Hartman, W. Song, A. Strominger, Black hole superradiance from Kerr/CFT, arXiv:0907.3477 [hep-th].

[20] M. Cvetic, F. Larsen, Greybody factors and charges in Kerr/CFT, JHEP 0909 (2009) 088, arXiv:0908.1136 [hep-th]; T. Hartman, W. Song, A. Strominger, Holographic derivation of Kerr-Newman scattering amplitudes for general charge and spin, JHEP 1003 (2010) 118, arXiv:0908.3909 [hep-th]; M. Becker, S. Cremonini, W. Schulgin, Extremal three-point correlators in Kerr/CFT, arXiv:1004.1174 [hep-th].

[21] J. Rasmussen, A near-NHEK/CFT correspondence, arXiv:1004.4773 [hep-th].

[22] A. Castro, A. Maloney, A. Strominger, Hidden conformal symmetry of the Kerr black hole, arXiv:1004.0996 [hep-th]; C. Krishnan, Hidden conformal symmetries of five-dimensional black holes, arXiv:1004.3537 [hep-th]; C.-M. Chen, J.-R. Sun, Hidden conformal symmetry of the Reissner-Nordstrøm black holes, arXiv:1004.3963 [hep-th]; Y.-Q. Wang, Y.-X. Liu, Hidden conformal symmetry of the Kerr-Newman black hole, arXiv:1004.4661 [hep-th]; B. Chen, J. Long, Real-time correlators and hidden conformal symmetry in Kerr/CFT correspondence, arXiv:1004.5039 [hep-th]; R. Li, M.-F. Li, J.-R. Ren, Entropy of Kaluza-Klein black hole from Kerr/CFT correspondence, arXiv:1004.5335 [hep-th]; C. Krishnan, Black hole vacua and rotation, arXiv:1005.1629 [hep-th].

[23] A. Strominger, Black hole entropy from near-horizon microstates, JHEP 9802 (1998) 009, arXiv:hep-th/9712251.

[24] M. Bañados, C. Teitelboim, J. Zanelli, The black hole in three dimensional space time, Phys. Rev. Lett. 69 (1992) 1849-1851, arXiv:hep-th/9204099.

[25] S. Carlip, Black hole entropy from conformal field theory in any dimension, Phys. Rev. Lett. 82 (1999) 2828-2831, arXiv:hep-th/9812013.

[26] S.N. Solodukhin, Conformal description of horizon's states, Phys. Lett. B454 (1999) 213-222, arXiv:hep-th/9812056.

[27] V.P. Frolov, K.S. Thorne, Renormalized stress-energy tensor near the horizon of a slowly evolving, rotating black hole, Phys. Rev. D39 (1989) 2125-2154. 